\title{
Folate levels measured by LC-MS/MS in patients with colorectal cancer treated with different leucovorin dosages
}

\author{
Helena Taflin • Yvonne Wettergren • Elisabeth Odin • \\ Kristoffer Derwinger
}

Received: 25 June 2014 / Accepted: 10 September 2014 / Published online: 20 September 2014

(C) The Author(s) 2014. This article is published with open access at Springerlink.com

\begin{abstract}
Purpose Calcium folinate (leucovorin), which is converted in vivo into biologically active folate, enhances the potency of 5-fluorouracil (5-FU)-based chemotherapy in colorectal cancer. A common dosage of leucovorin in adjuvant and palliative settings is $60 \mathrm{mg} / \mathrm{m}^{2}$. The aim was to determine the levels of tetrahydrofolate (THF), 5,10-methylenetetrahydrofolate (methyleneTHF), and 5-methyltetrahydrofolate (methylTHF) in tumour and mucosa of colorectal cancer patients who received different dosages of leucovorin intravenously at time of surgery.

Methods Eighty patients scheduled for colorectal resection with indication of colorectal cancer were randomised into four groups to receive leucovorin at $0,60,200$, or $500 \mathrm{mg} / \mathrm{m}^{2}$, respectively. Blood samples were taken 10 and $30 \mathrm{~min}$ after leucovorin administration. Biopsy samples from tumour and mucosa were collected and snap-frozen at surgery. The levels of THF, methyleneTHF, and methylTHF in tumour and mucosa were assessed by liquid chromatography electrospray ionisation tandem mass spectrometry (LC-MS/MS) and the results were related to clinical diagnosis and therapeutic regimens.

Results The folate levels in tissue revealed extensive inter-individual variability. The mean methyleneTHF value for the four treatment groups were 880, 1,769, 3,024 and $3,723 \mathrm{pmol} / \mathrm{g}_{\mathrm{ww}}$. Only half of the patients who received $60 \mathrm{mg} / \mathrm{m}^{2}$ leucovorin had higher levels of methyleneTHF in tumour than patients who received $0 \mathrm{mg} / \mathrm{m}^{2}$ leucovorin.
\end{abstract}

H. Taflin $(\bowtie) \cdot$ Y. Wettergren $\cdot$ E. Odin $\cdot$ K. Derwinger

Department of Surgery, Institute of Clinical Sciences,

The Sahlgrenska Academy at University of Gothenburg,

The Sahlgrenska University Hospital/Östra,

41685 Gōteborg, Sweden

e-mail: helena.taflin@vgregion.se
Rectal cancer patients had significantly lower levels of methyleneTHF compared with colon cancer patients.

Conclusions There was a large inter-patient variability of tissue folate levels in colorectal cancer patients after supplementation with leucovorin at standardised dosage. High leucovorin doses were needed to exceed baseline methyleneTHF values, especially in rectal cancer patients. The results indicate that the standardised leucovorin dose may be insufficient to attain the full antitumour effect of 5-FU. Further studies are needed to establish whether higher dosage yields a better treatment response.

Keywords Colorectal cancer - Leucovorin · 5-FU-based treatment $\cdot$ Methylenetetrahydrofolate $\cdot$ Tetrahydrofolate . Formyltetrahydrofolate

\section{Introduction}

In 2012, colorectal cancer was the second most diagnosed cancer in Europe after breast cancer. Colorectal cancer was also responsible for the second highest number of cancerrelated deaths after lung cancer [1]. Currently, the only curative therapy for patients with colorectal cancer is surgery with radical removal of the tumour. When the cancer is limited to the bowel wall, surgery itself normally attains the desired oncologic outcome. In cases of lymph node involvement, i.e. stage III disease, there is a high risk of tumour recurrence. It has been confirmed in several studies that a 5-fluorouracil (5-FU)-based chemotherapy regimen improves both overall and disease-free survival for patients with stage III disease [2-4]. Considering the numbers of patients and administered treatments, it is of great importance to find methods to tailor or at least optimise the therapy. However, even patients with high-risk profiles, 
such as poorly differentiated tumours without lymph node metastasis, could benefit from additional treatment [5] [6]. In this post-operative setting, the term adjuvant treatment is commonly used. 5-FU-based chemotherapy has also been shown to prolong overall survival in palliative settings, i.e. for patients with confirmed distant metastasis [7].

5-Fluorouracil was developed in the 1950s by Charles Heidelberger, who discovered that rat hepatomas were consuming the pyrimidine uracil more rapidly than normal rat liver tissue [8]. Thus, uracil was identified as a target molecule for chemotherapy. 5-FU is an analogue of uracil in which the hydrogen at position 5 is replaced by fluorine. Using the same mechanism to enter the cell as uracil, the 5-FU molecule is converted into the active metabolite 5-fluoro-2'-deoxyuridine 5 -monophosphate (FdUMP), which forms an inhibitory ternary complex with thymidylate synthase (TS) and 5,10-methylenetetrahydrofolate (methyleneTHF). This results in the inhibition of thymidylate synthesis and impairment of both DNA synthesis and DNA repair. The greatest impact is on cells that are rapidly dividing, such as tumour epithelial cells.

The response rate of colorectal tumours to 5-FU monotherapy is only around $10 \%$. By adding the stable calcium salt of 5-formyltetrahydrofolic acid (Calciumfolinate), which is converted in the liver into methyleneTHF, the tumour response rate can be improved to $21 \%$, as has been shown in a meta-analysis [9]. The Nordic FLV therapy, which is a combination of 5-FU and leucovorin (REF 12) that was introduced in the 1990s, is still the cornerstone of both adjuvant and palliative treatments for colorectal cancer in Nordic countries. The standard dosage is $500 \mathrm{mg} /$ $\mathrm{m}^{2} 5$-FU plus $60 \mathrm{mg} / \mathrm{m}^{2}$ leucovorin in the form of calciumfolinate, administered as an intravenous infusion 2 days in a row. The 2-day treatment is followed by a pause for 12 days. The standard protocol is usually 6 months long. The regimen has been duly updated with the incorporation of novel drugs, such as oxaliplatin and antibodies into more effective combination therapies. Although it is a well established regimen, the evidence for the leucovorin dosage used is rather limited. Different regimens used in clinical practice worldwide apply levels of leucovorin that range from 20 to $500 \mathrm{mg} / \mathrm{m}^{2}$.

Leucovorin has no intrinsic antitumour effect but it enhances the effect of 5-FU by providing the cofactor methyleneTHF in abundance and by stabilising the ternary complex [10]. However, leucovorin must first be converted in two steps into methyleneTHF, which is the active metabolite. This requirement for metabolic activation may result in inter-individual differences in uptake, thereby compromising the benefit gained from the addition of leucovorin in some of the patients. The leucovorin metabolism pathway has been described by Priest and colleagues [11].
The impressive advances that have been made in genetics and metabolite measurements (metabolomics) provide new possibilities for advanced studies of the folate metabolism and facilitate a better understanding of related cellular mechanisms. Our research group presented in 2012 an LC-MS/MS method that is sufficiently sensitive to separate and quantify different forms of folate [12]. Thus, the actual concentration of methyleneTHF, and not only of total folates, can now be measured in tissue samples. As evidenced by our recent findings, using the novel method, there is a significant variability in the folate levels in tumour and mucosa tissues between patients [13].

The aim of the present study was to determine the levels of different folate forms in tumour and mucosa tissue of patients with colorectal cancer who received different dosages of leucovorin intravenously at the time of surgery. The folate levels were related to the clinical diagnosis and therapeutic regimens used.

\section{Patients and methods}

The study was approved by the Regional Ethics Committee in Gothenburg (EPN). Eighty patients scheduled for a colorectal resection with a cancer indication were enrolled in the study between January 2011 and January 2012. All patients gave their written informed consent. The preoperative exclusion criteria were patient inability to understand the study information or inability to provide true informed consent. There were no other exclusion criteria (such as ASA-class, renal function or pre-operative tumour stage). The patients were pre-operatively randomised into four groups; the first served as control group and received no leucovorin. Groups 2, 3, and 4 received 60, 200, and $500 \mathrm{mg} / \mathrm{m}^{2}$ leucovorin, respectively, administered intravenously at the initiation of general anaesthesia. The leucovorin was manufactured in the form of calcium folinate (dlleucovorin) supported by Teva Sweden AB Helsingborg. The surgeon was blinded to the dosage given. The patients were otherwise treated in accordance with normal routines and guidelines.

During surgery, at the time of removal of the surgical specimen, the research nurse collected fresh tissue samples from both the tumour and macroscopically normalappearing mucosa located $10 \mathrm{~cm}$ from the tumour. The biopsies were snap-frozen in liquid nitrogen and stored at $-80{ }^{\circ} \mathrm{C}$ until used. The pathology department assessed the specimens and provided tumour staging data. Based on the routine pathology reports, four patients were excluded from the study because the analysis revealed a lack of adenocarcinoma tissue; two patients had an obstruction related to diverticulitis, one had a squamous epithelial cancer, and one had a non-malignant adenoma. During 
analysis of blood samples, we discovered that one patient in treatment group two had received a leucovorin dose that was not according to the protocol and this patient is also excluded from the study. The main assessment was of the folate levels in the mucosa and tumour tissues in relation to treatment group. Clinical and pathology data regarding diagnosis, tumour differentiation and stage, and pre-operative treatment regimen were retrieved to assess the different groups and enable a better understanding of the factors that might influence treatment responses.

\section{Folate analyses}

A liquid chromatography electrospray ionisation tandem mass spectrometry (LC-MS/MS) method was used to evaluate the levels of the folate derivatives, tetrahydrofolate (THF), methyleneTHF, and 5-methyltetrahydrofolate (methylTHF) in tumour tissue and adjacent mucosa, separately [12].). The LC-MS/MS analyses were performed on a waters 2795 LC separation module coupled to a waters micromass Quattro Triple-Quadrupole MS system with an electrospray ionisation (ESI) source. Folates were detected and quantified using positive electrospray. The separation of folates was performed using an Atlantis $\mathrm{dC}_{18} 3 \mu \mathrm{m}, 2.1 * 100 \mathrm{~mm}$ column (waters) together with the guard column Atlantis $\mathrm{dC}_{18}, 3 \mu \mathrm{m}, 2.1 * 10 \mathrm{~mm}$. The mobile phase consisting of eluent A $(0.1 \%$ of acetic acid in water) and eluent B (0.1\% acetic acid in acetonitrile) was used. The extracted ions following MRM transitions were monitored at $\mathrm{m} / \mathrm{z} 446 \rightarrow 299$ for THF, $\mathrm{m} / \mathrm{z}$ $458 \rightarrow 311$ for methyleneTHF, $\mathrm{m} / \mathrm{z} 460 \rightarrow 313$ for methylTHF, and $\mathrm{m} / \mathrm{z} 459 \rightarrow 312$ for Tomudex (IS). On the day of sample analysis, extraction buffer was prepared containing $50 \mathrm{mM}$ phosphate buffer, $\mathrm{pH} 7.0,1 \%$ ascorbate, and $0.1 \%$ $\beta$-mercaptopropanol. The tissue was weighed and placed in an Eppendorf vial and a $10 \times$ volume of extraction buffer was added. Homogenisation was performed using a TissueLyzer (two disruption steps at $25 \mathrm{~Hz}$ for $2.5 \mathrm{~min}$ ). Tomudex was used as an intern standard. After deconjugation, protein precipitation, centrifugation, and ultrafiltration (30 min at $21,500 \times g$ at $20{ }^{\circ} \mathrm{C}$ ) were performed. The solution at the bottom of the test tube was used for the LC-MS/MS analysis.

Calibration graphs were constructed by plotting the peak area ratio of each compound to internal standards against concentration. The standards and samples were processed using the QuanLynx quantitative processing tool in MassLynx (Waters Corp., Milford, MA, USA). Intra-batch variability was determined by analysing tissue Q-samples at low, medium, and high concentrations on the same day. Inter-assay variability was determined by analysing low, medium, and high concentration samples on four separate days. The relative standard deviation (RSD) ranged from
2 to $7 \%$ for all analyses, and the variability over 4 days ranged from 3 to $14 \%$ for all analyses. The accuracy of the method was determined by estimating the recovery by adding known amounts of the standard to a sample. The average recoveries were 98,87 , and $93 \%$ for THF, methyleneTHF, and methylTHF, respectively [12].

The levels of THF, methyleneTHF, and methylTHF in each sample were expressed as $\mathrm{pmol} / \mathrm{g}$ wet-weight (pmol/ $\mathrm{g}_{\mathrm{ww}}$ ). Due to the known interconversion of methyleneTHF and THF, the sum of the concentrations of these two folates were also calculated [14].

The plasma samples were frozen, stored, and shipped at $-80{ }^{\circ} \mathrm{C}$ to Charles River Laboratories, UK, where the plasma concentrations of methyleneTHF, THF, methylTHF, and formyl-THF were analysed using a validated LC-MS/ MS method.

\section{Statistical analyses}

The JMP 11.0/SAS software (SAS Institute Inc., Cary, NC, USA) was used for the statistical analyses. Nonparametric tests; Mann-Whitney/Kruskal-Wallis, and matched-pair analyses (Wilcoxon Signed Rank test) were used to examine differences between the groups. Also presented are descriptive statistics with mean or median values and measures of dispersion, as appropriate. The significance level was set at $95 \%$.

\section{Results}

Patients and treatment regimens

Based on clinical diagnosis, 38 patients had colon cancer, 37 had rectal cancer, and three patients had cancer in both the colon and rectum synchronously. The latter three patients were excluded from the statistical analyses of folate levels according to tumour location. The demographic, clinical, and pathologic data are shown in Table 1. There were no significant differences regarding age, gender, tumour location, tumour stage, tumour differentiation, or lymph status between the four groups. The mean times between administration of leucovorin and time of biopsy sampling, as well as ranges for the three groups are shown in Table 1.

As shown, no statistical differences between the times from leucovorin administration to biopsy sampling were seen. However, a difference regarding pre-operative treatment was noted; in the control group, there was no patient with rectal cancer who had been given pre-operative radiation treatment or neoadjuvant treatment. Regarding safety, the administration of the drug was associated with temporary red cheeks in one patient (given $200 \mathrm{mg} / \mathrm{m}^{2}$ leucovorin) 
Table 1 Clinicopathological characteristics of the colorectal cancer patients sub grouped by leucovorin dosage

\begin{tabular}{|c|c|c|c|c|}
\hline \multirow[t]{2}{*}{ Parameter } & \multicolumn{4}{|c|}{ Leucovorin dosage $(n)$} \\
\hline & $0 \mathrm{mg} / \mathrm{m}^{2}[18]$ & $60 \mathrm{mg} / \mathrm{m}^{2}[18]$ & $200 \mathrm{mg} / \mathrm{m}^{2}[19]$ & $500 \mathrm{mg} / \mathrm{m}^{2}[20]$ \\
\hline \multicolumn{5}{|l|}{ Age (years) } \\
\hline Median & 73 & 65.6 & 70 & 75.5 \\
\hline Range & $43-89$ & $42-87$ & $37-89$ & $37-87$ \\
\hline \multicolumn{5}{|l|}{ Sex } \\
\hline Male & 11 & 12 & 7 & 9 \\
\hline Female & 7 & 6 & 12 & 11 \\
\hline \multicolumn{5}{|l|}{ Tumour location } \\
\hline Colon & 9 & 10 & 8 & 11 \\
\hline Rectum & 8 & 8 & 10 & 8 \\
\hline Colon + rectum & 1 & 0 & 1 & 1 \\
\hline \multicolumn{5}{|l|}{ Primary tumour stage } \\
\hline 1 & 0 & 1 & 1 & 3 \\
\hline 2 & 7 & 9 & 9 & 7 \\
\hline 3 & 9 & 8 & 7 & 8 \\
\hline 4 & 4 & 0 & 2 & 2 \\
\hline Data missing & 1 & 0 & 0 & 0 \\
\hline \multicolumn{5}{|l|}{ Tumour differentiation } \\
\hline Well & 0 & 0 & 0 & 0 \\
\hline Moderate & 14 & 13 & 11 & 12 \\
\hline Poor & 3 & 2 & 6 & 6 \\
\hline Mucinous & 0 & 3 & 2 & 2 \\
\hline Data missing & 1 & 0 & 0 & 0 \\
\hline \multicolumn{5}{|l|}{ Pre-operative radiation } \\
\hline Short-term & 0 & 6 & 5 & 2 \\
\hline Long-term ${ }^{a}$ & 0 & 0 & 2 & 1 \\
\hline Short + long-term ${ }^{\mathrm{a}}$ & 0 & 1 & 7 & 3 \\
\hline $\begin{array}{l}\text { Median time (min) leucovorin } \\
\text { administration-biopsy sampling } \\
\text { (mean range): }\end{array}$ & & $170(65-285)$ & $165(72-457)$ & $163(65-555)$ \\
\hline
\end{tabular}

a Neoadjuvant long-term radiation/chemotherapy

and a short temporary hypotension reaction in one patient (given $500 \mathrm{mg} / \mathrm{m}^{2}$ ). These episodes were considered as adverse events. In none of the cases did the adverse reaction lead to any change in the operating procedure.

Folate levels in tumours and mucosa

The mean levels of methyleneTHF, THF, and methylTHF were analysed in both tumour and mucosa tissues obtained from patients of each treatment group (Table 2). The mean level of each folate increased with increasing dosage of leucovorin and showed a large inter-patient variation in all treatment groups. The folate levels differed significantly between the mucosa and tumour tissues and were generally lower in the mucosa of the control group. Patients who received 60 or $200 \mathrm{mg} / \mathrm{m}^{2}$ leucovorin had significantly higher mean levels of methyleneTHF in their tumours, as compared to the levels in their mucosal samples. After treatment with $500 \mathrm{mg} / \mathrm{m}^{2}$ leucovorin, the difference in methyleneTHF level between the tumour and mucosa samples was no longer statistically significant. The same pattern was seen when the THF concentration or the sum of methyleneTHF and THF were analysed. No significant differences in the levels of methylTHF were seen between the tumour and mucosa samples in any of the treatment groups. However, in contrast to the other folates, the methylTHF level in mucosa of the control group was significantly higher compared to the level in tumour tissue.

Folate levels and tumour location

There were differences between the folate levels in colonic and rectal tumours according to treatment doses. For all 
Table 2 Comparison of mean \pm SD folate levels in tumour and mucosa tissues of the colorectal cancer patients

\begin{tabular}{|c|c|c|c|c|c|}
\hline \multirow[t]{2}{*}{ Folate form } & \multirow[t]{2}{*}{ Tissue type } & \multicolumn{4}{|c|}{ Leucovorin dosage $(n)$} \\
\hline & & $0 \mathrm{mg} / \mathrm{m}^{2}[18]$ & $60 \mathrm{mg} / \mathrm{m}^{2}[18]$ & $200 \mathrm{mg} / \mathrm{m}^{2}[19]$ & $500 \mathrm{mg} / \mathrm{m}^{2}[20]$ \\
\hline \multirow[t]{2}{*}{ THF } & Tumour & $136 \pm 88$ & $249 \pm 97$ & $490 \pm 353$ & $543 \pm 279$ \\
\hline & Mucosa & $103 \pm 59$ & $210 \pm 96$ & $289 \pm 153$ & $455 \pm 282$ \\
\hline$P$ value & & 0.030 & 0.091 & $<0.0001$ & 0.12 \\
\hline \multirow[t]{2}{*}{ MethyleneTHF } & Tumour & $880 \pm 412$ & $1,769 \pm 818$ & $3,024 \pm 1,941$ & $3,773 \pm 1,425$ \\
\hline & Mucosa & $669 \pm 221$ & $1,377 \pm 470$ & $1,883 \pm 471$ & $3,062 \pm 1,445$ \\
\hline$P$ value & & 0.0016 & 0.022 & 0.0003 & 0.090 \\
\hline \multirow[t]{2}{*}{ MethyleneTHF + THF } & Tumour & $1,016 \pm 475$ & $2,018 \pm 888$ & $3,514 \pm 2,109$ & $4,266 \pm 1,563$ \\
\hline & Mucosa & $772 \pm 265$ & $1,587 \pm 536$ & $2,173 \pm 461$ & $3,517 \pm 1,607$ \\
\hline$P$ value & & 0.010 & 0.018 & 0.0002 & 0.070 \\
\hline \multirow[t]{2}{*}{ MethylTHF } & Tumour & $141 \pm 86$ & $1,056 \pm 348$ & $2,544 \pm 959$ & $4,129 \pm 1,293$ \\
\hline & Mucosa & $189 \pm 111$ & $1,066 \pm 384$ & $2,295 \pm 501$ & $4,095 \pm 2,093$ \\
\hline$P$ value & & 0.0047 & 0.93 & 0.46 & 0.49 \\
\hline \multicolumn{6}{|l|}{ Leucovorin (mean/range) ${ }^{\mathrm{a}}$} \\
\hline $10 \mathrm{~min}$ & & Non applicable & $11,377 \pm 2,225$ & $30,900 \pm 6,176$ & $93,625 \pm 1,872$ \\
\hline $30 \mathrm{~min}$ & & & $8,199 \pm 1,238$ & $27,684 \pm 41,013$ & $69,445 \pm 9,172$ \\
\hline
\end{tabular}

Folate levels in $\mathrm{pmol} / \mathrm{g}_{\mathrm{ww}}$

$\mu \mathrm{g} / \mathrm{L}$ plasma

$P$ value by Wilcoxon Signed Rank test

${ }^{a}$ Concentration in plasma

Table 3 Comparison of mean \pm SD folate levels in tumour tissues of the colorectal cancer patients by tumour location

\begin{tabular}{|c|c|c|c|c|c|}
\hline \multirow[t]{2}{*}{ Folate form } & \multirow[t]{2}{*}{ Tumour location } & \multicolumn{4}{|c|}{ Leucovorin dosage $(n)$} \\
\hline & & $0 \mathrm{mg} / \mathrm{m}^{2}[17]$ & $60 \mathrm{mg} / \mathrm{m}^{2}[18]$ & $200 \mathrm{mg} / \mathrm{m}^{2}[18]$ & $500 \mathrm{mg} / \mathrm{m}^{2}[19]$ \\
\hline \multirow[t]{2}{*}{ THF } & Colon & $143 \pm 62$ & $306 \pm 79$ & $508 \pm 240$ & $522 \pm 164$ \\
\hline & Rectum & $139 \pm 121$ & $178 \pm 67$ & $497 \pm 446$ & $494 \pm 317$ \\
\hline$P$ value & & 0.41 & 0.0067 & 0.18 & 0.24 \\
\hline \multirow[t]{2}{*}{ MethyleneTHF } & Colon & $1,016 \pm 482$ & $2,214 \pm 779$ & $4,207 \pm 2,590$ & $4,222 \pm 1,285$ \\
\hline & Rectum & $747 \pm 230$ & $1,213 \pm 454$ & $2,162 \pm 406$ & $3,222 \pm 1,530$ \\
\hline$P$ value & & 0.26 & 0.024 & 0.0029 & 0.066 \\
\hline \multirow[t]{2}{*}{ MethyleneTHF + THF } & Colon & $1,159 \pm 537$ & $2,520 \pm 826$ & $4,715 \pm 2,821$ & $4,744 \pm 1,397$ \\
\hline & Rectum & $886 \pm 328$ & $1,391 \pm 477$ & $2,659 \pm 696$ & $3,716 \pm 1,726$ \\
\hline$P$ value & & 0.26 & 0.0088 & 0.019 & 0.094 \\
\hline \multirow[t]{2}{*}{ MethylTHF } & Colon & $162 \pm 99$ & $1,257 \pm 201$ & $2,331 \pm 757$ & $4,022 \pm 1,551$ \\
\hline & Rectum & $123 \pm 63$ & $806 \pm 338$ & $2,795 \pm 1,100$ & $4,163 \pm 1,119$ \\
\hline$P$ value & & 0.46 & 0.0067 & 0.56 & 0.54 \\
\hline
\end{tabular}

Folate levels in $\mathrm{pmol} / \mathrm{g}_{\mathrm{ww}}$

$P$ value by Kruskal-Wallis test (2-sample Test)

treatments groups, the mean methyleneTHF levels in the rectal tumours were significantly lower than those in the colonic tumours (Table 3; Figs. 1, 2). The difference was significant in the groups that received 60 or $200 \mathrm{mg} / \mathrm{m}^{2}$ leucovorin. The THF level was significantly lower in rectal tumours of patients who received $60 \mathrm{mg} / \mathrm{m}^{2}$. As shown in
Table 3, the methyleneTHF + THF levels were generally low in rectal, compared to colon, tumours. In contrast, the methylTHF levels were higher in rectal tumour tissues of patients who were treated with leucovorin, and a significantly higher level was seen after treatment with $60 \mathrm{mg} /$ $\mathrm{m}^{2}$. As shown in Fig. 2, only $10(50 \%)$ of the patients 


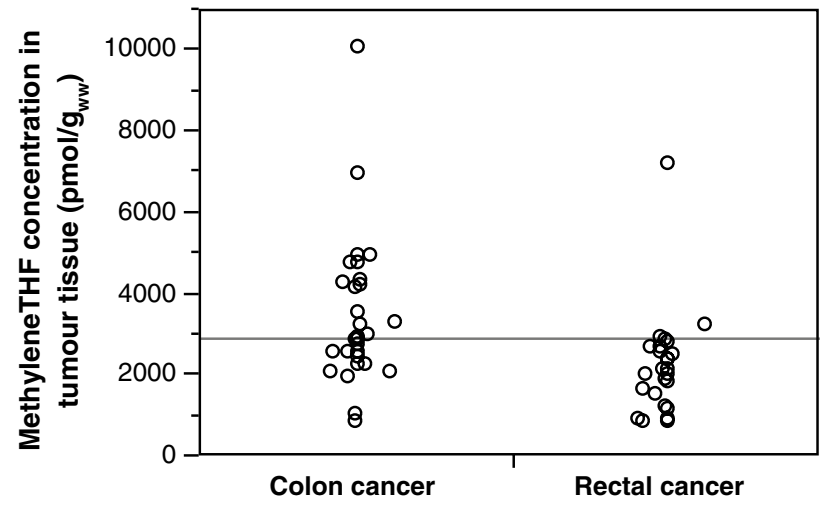

Fig. 1 Comparison of the methyleneTHF concentration in tumour tissue of patients with colon $(n=29)$ or rectal $(n=28)$ cancer after FLV treatment. Each circle represents an individual patient. As shown, patients with rectal cancer had lower levels of methyleneTHF in their tumours. The horizontal line represents the grand mean

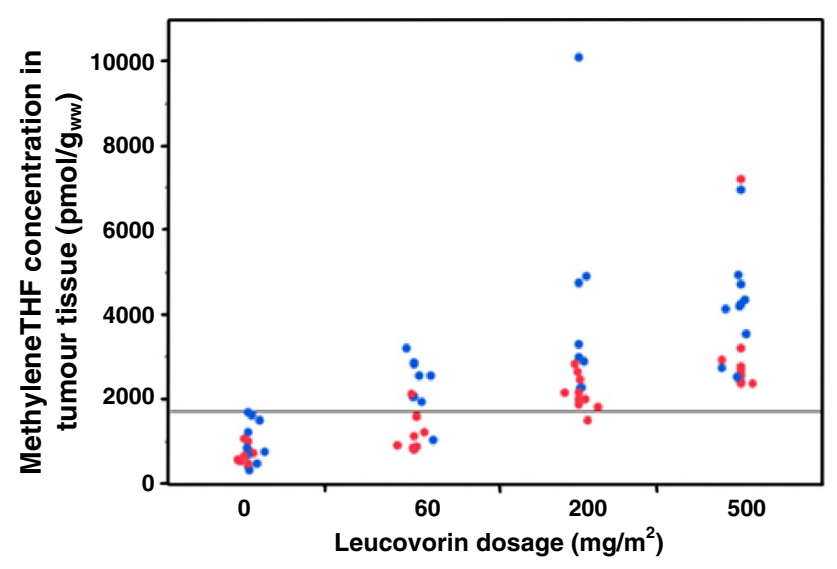

Fig. 2 Comparison of the methyleneTHF levels in tumour tissue of patients with colon or rectal cancer after supplementation with 0,60 , 200 , or $500 \mathrm{mg} / \mathrm{m}^{2}$ leucovorin in combination with 5 -FU. Individual patients with colon cancer are represented by blue dots, rectal cancer patients with red dots. The horizontal line marks the highest methyleneTHF concentration found in the control patients $(1,714 \mathrm{pmol} /$ $\left.\mathrm{g}_{\mathrm{ww}}\right)$. As shown, the tumoural methyleneTHF levels in rectal cancer patients were generally lower than in colon cancer patients after treatment with leucovorin

given $60 \mathrm{mg} / \mathrm{m}^{2}$ leucovorin achieved a methyleneTHF level in their tumour tissues that was above the highest value of any patient in the control group $\left(1,714 \mathrm{pmol} / \mathrm{g}_{\mathrm{ww}}\right)$. At $200 \mathrm{mg} / \mathrm{m}^{2}$ leucovorin, all patients, except one, reached methyleneTHF levels $>1,714 \mathrm{pmol} / \mathrm{g}_{\mathrm{ww}}$, and at $500 \mathrm{mg} / \mathrm{m}^{2}$ leucovorin, all patients had methyleneTHF levels above the level of the controls. Data were weighted according to time to vessel ligation, which was a parameter suspected to affect the tissue folate levels. However, this did not affect the significant differences between colon and rectal tumour tissue.
Blood analysis

A strong correlation was noted between the levels of leucovorin in blood samples collected 10 and $30 \mathrm{~min}$ after the given dose of calcium folinate and the levels of the different folate forms in both tumour and mucosa samples. However, there was no correlation between the blood levels of leucovorin and levels of folate in tissue in relation to the administered dosage of the drug.

\section{Discussion}

The role of leucovorin in 5-FU-based chemotherapy is to increase the level of the cofactor methyleneTHF needed to stabilise the ternary complex consisting of FdUMP, methyleneTHF, and TS in tumour cells. Thereby, the production of thymidine is inhibited leading to an impaired DNA synthesis and DNA repair. The continuous development of assessment techniques and new biochemical methods provide tools to study different metabolites in more sophisticated ways. In the present study, a sensitive LC-MS/MS method was used to analyse metabolically active folate metabolites, including methyleneTHF.

A notable finding of the study was that only $50 \%$ of the patients who received the common dose of $60 \mathrm{mg} / \mathrm{m}^{2}$ leucovorin achieved methyleneTHF levels in the tumour tissue that were higher than those detected in patients of the control group. In contrast, in the group that received $200 \mathrm{mg} /$ $\mathrm{m}^{2}$ leucovorin, only one patient did not reach the highest methyleneTHF level of the controls. Furthermore, in the group that received $500 \mathrm{mg} / \mathrm{m}^{2}$ leucovorin, all patients had a methyleneTHF level well above the controls. This indicates that in a large number of patients low levels of folates accumulated in the tumour, possible because of limited folate polyglutamylation. Another important finding was the significant difference in methyleneTHF and THF levels between the colorectal tumour tissue and the macroscopically normal-appearing mucosa (Table 2). This difference was observed in patients who had not received any treatment and was even more pronounced in those patients who received leucovorin at a dosage of 60 or $200 \mathrm{mg} / \mathrm{m}^{2}$.

The study raises the question whether the commonly used dosage of leucovorin might result in a sub-optimal concentration of methyleneTHF in the tumour tissue to provide an optimal effect of 5-FU treatment. If this is the case, several patients with colorectal cancer might be receiving inadequate treatment and may benefit from leucovorin concentrations as high as $200-500 \mathrm{mg} / \mathrm{m}^{2}$. Similar conclusions were drawn by Schlemmer et al. [15] in a study published in 2008, which showed significant higher values regarding reduced folates in tumour tissues as well as in liver metastasis when doses of 200 and $500 \mathrm{mg} / \mathrm{m}^{2}$ were used. 
Adding to the complexity, there was a high inter-individual variation in the methyleneTHF levels in the tumours (Fig. 2). Polymorphisms in genes that code for folate-associated enzymes, such as methylenetetrahydrofolate reductase, could be a part of the explanation for this phenomenon [16-19]. Different activity of the enzymes needed in conversion of leucovorin to methyleneTHF could also play a role, as indeed could different starting levels of the tissue folates. Therefore, it is difficult to predict the levels of tissue folates that will be reached in an individual patient, in response to a given leucovorin dose based solely on the body surface area. However, since leucovorin is not considered to be a toxic substance and only a few and mild adverse events were reported in the present study, further studies with high leucovorin supplementation levels $\left(200-500 \mathrm{mg} / \mathrm{m}^{2}\right)$ could be reasonably pursued. Hypothetically, high doses of leucovorin would make an abundance of methyleneTHF available for ternary complex formation between methyleneTHF, FdUMP, and TS, despite possible rate-limiting steps or local folate deficiencies. The results further showed clear differences in the folate levels in relation to tumour location. The methyleneTHF and THF + methyleneTHF levels differed significantly between patients with colon and rectal cancer (Table 3). For all treatment groups, the levels were lower for rectal cancers. No significant difference was seen between patients treated or not treated with neoadjuvant therapy.

Post-operative adjuvant chemotherapy was previously less established for cases of rectal cancer than for cases of colon cancer, but is now recommended in the Swedish national guidelines in selected cases. Based on the results of our study, it appears that the administered concentration of leucovorin in the standard treatment is inadequate for patients with rectal cancer in that it yields a clinically insufficient concentration of methyleneTHF in the tumour tissue. This finding may explain why the evidence for a beneficial application of adjuvant treatment with 5-FUbased chemotherapy has not been as clear in rectal cancer as they are in colon cancer [20,21].

The present study draws attention to several issues. The findings raise the question as to whether previous assumptions made regarding leucovorin dosages are correct. It also shows that new techniques and advances in associated scientific areas make revaluation of previously studied subjects both worthwhile and important. As the present study was limited in terms of the numbers of patients, the results needs to be confirmed in a larger study. Among the strengths of the study are the broad inclusion criteria, reflecting clinical reality, and the randomisation of the patients to the treatment groups. The randomisation should negate selection bias, although skewing was noted between patient groups in terms of neoadjuvant treatment; no patient in the control group received any pre-operative radiotherapy. Because a high turnover of folates might be required during repair of radiation-damaged tissue, the mean folate level at base line could be anticipated to be lower if the rectal cancer patients of the control group had been subjected to radiotherapy. However, we could not detect any differences in the folate levels between patients treated or not treated with radiotherapy. Furthermore, both higher (methylTHF) and lower (methyleneTHF) folate levels were found in rectal tumours as compared to colon tumours, after leucovorin supplementation. Thus, there seems to be inherent differences in the response to leucovorin treatment between rectal and colon cancer.

The possibility exists that the difference in folate levels noted is a systematic bias due to treatment or surgical issues, including differences in the time passed after vessel ligation until biopsy sampling. However, this potential confounder was tested for by including the time to vessel ligation in the statistical analysis and did not significantly affect the results. In a recent study, Sadahiro et al. showed that leucovorin administration significantly increased the reduced folate levels in colorectal cancer tissue and adjacent mucosa. The increase lasted until $18 \mathrm{~h}$ for mucosa and $12 \mathrm{~h}$ for colorectal cancer tissue [22]. However, in a clinical setting, the time span from the leucovorin administration until infusion of chemotherapy is usually no longer than 30-90 min. It can also be debated as to whether the fact that a tumour is a very heterogenic tissue has an important influence on the measured folate concentrations depending on how the biopsies are collected. In the present study, we have tried to address this problem by having the same two research nurses collecting tissues in a standardised way. The data suggest that our results are comparable to previous results reported in the literature [14, 23, 24].

A last and major challenge is the difficulty of extrapolating the findings into adjuvant treatment settings, as any visible tumour tissue has been removed by the time adjuvant treatment starts. Thus, the treatment effect will be exerted on normal tissues and, hopefully, on remaining circulating tumour cells or cell aggregations. The nature of this issue itself presents challenges that need to be resolved in order to achieve treatment improvements.

\section{Conclusions}

The results of this study showed a large inter-patient variability of folate levels in tumour and mucosa tissue of colorectal patients after supplementation with leucovorin at the standardised dosage. The low levels of methyleneTHF were most prominent in rectal cancer patients, where high leucovorin doses were needed to exceed baseline methyleneTHF values in tumour. The results of this study indicate that the standardised dose may be sub-optimal since the achieved 
concentration of methyleneTHF in tissues of the patients may be insufficient to get an optimal antitumour effect of 5-FU. On going studies will establish if a higher concentration of leucovorin yields a better treatment response.

Acknowledgments We acknowledge the Swedish Cancer Society, grants from the Swedish state under the LUA-ALF agreement, the Assar Gabrielsson Foundation for Cancer Research, the Gothenburg Medical Society, The Lions Cancer Foundation West, and the Bror och Anna-Lisa Björnsson Foundation for financial support. We thank J. Flach, and M. Åkerström for technical assistance, A-L. Helminen, H. Björkquist, and L. Munro for collecting patients' samples, and L. Munro and B. Sjöberg for work on the clinical database.

Open Access This article is distributed under the terms of the Creative Commons Attribution License which permits any use, distribution, and reproduction in any medium, provided the original author(s) and the source are credited.

\section{References}

1. Ferlay $\mathbf{J}$ et al (2013) Cancer incidence and mortality patterns in Europe: estimates for 40 countries in 2012. Eur J Cancer 49(6):1374-1403

2. Wolmark $\mathrm{N}$ et al (1993) The benefit of leucovorin-modulated fluorouracil as postoperative adjuvant therapy for primary colon cancer: results from National Surgical Adjuvant Breast and Bowel Project protocol C-03. J Clin Oncol 11(10):1879-1887

3. (1995) Efficacy of adjuvant fluorouracil and folinic acid in colon cancer. International Multicentre Pooled Analysis of Colon Cancer Trials (IMPACT) investigators. Lancet 345(8955): 939-944

4. Golfinopoulos V et al (2007) Survival and disease-progression benefits with treatment regimens for advanced colorectal cancer: a meta-analysis. Lancet Oncol 8(10):898-911

5. Wilkinson NW et al (2010) Long-term survival results of surgery alone versus surgery plus 5-fluorouracil and leucovorin for stage II and stage III colon cancer: pooled analysis of NSABP C-01 through C-05. A baseline from which to compare modern adjuvant trials. Ann Surg Oncol 17(4):959-966

6. Lombardi L et al (2010) Adjuvant colon cancer chemotherapy: where we are and where we'll go. Cancer Treat Rev 36(Suppl 3):S34-S41

7. Lucas AS, O'Neil BH, Goldberg RM (2011) A decade of advances in cytotoxic chemotherapy for metastatic colorectal cancer. Clin Colorectal Cancer 10(4):238-244

8. Heidelberger C, Danenberg PV, Moran RG (1983) Fluorinated pyrimidines and their nucleosides. Adv Enzymol Relat Areas Mol Biol 54:58-119
9. Thirion P et al (2004) Modulation of fluorouracil by leucovorin in patients with advanced colorectal cancer: an updated meta-analysis. J Clin Oncol 22(18):3766-3775

10. Longley DB, Harkin DP, Johnston PG (2003) 5-Fluorouracil: mechanisms of action and clinical strategies. Nat Rev Cancer 3(5):330-338

11. Priest DG et al (1991) Pharmacokinetics of leucovorin metabolites in human plasma as a function of dose administered orally and intravenously. J Natl Cancer Inst 83(24):1806-1812

12. Odin E et al (2013) Determination of reduced folates in tumor and adjacent mucosa of colorectal cancer patients using LC-MS/ MS. Biomed Chromatogr 27(4):487-495

13. Taflin $\mathrm{H}$ et al (2014) Folate levels and polymorphisms in the genes MTHFR, MTR, and TS in colorectal cancer. Clin Med Insights Oncol 8:15-20

14. Spears CP, Gustavsson BG (1988) Methods for thymidylate synthase pharmacodynamics: serial biopsy, free and total TS, FdUMP and dUMP, and H4PteGlu and $\mathrm{CH} 2-\mathrm{H} 4$ PteGlu assays. Adv Exp Med Biol 244:97-106

15. Schlemmer M et al (2008) Tissue levels of reduced folates in patients with colorectal carcinoma after infusion of folinic acid at various dose levels. Clin Cancer Res 14(23):7930-7934

16. Taflin $\mathrm{H}$ et al (2011) Gene polymorphisms MTHFRC677T and MTRA2756G as predictive factors in adjuvant chemotherapy for stage III colorectal cancer. Anticancer Res 31(9):3057-3062

17. De Mattia E, Toffoli G (2009) C677T and A1298C MTHFR polymorphisms, a challenge for antifolate and fluoropyrimidine-based therapy personalisation. Eur J Cancer 45(8):1333-1351

18. Cohen V et al (2003) Methylenetetrahydrofolate reductase polymorphism in advanced colorectal cancer: a novel genomic predictor of clinical response to fluoropyrimidine-based chemotherapy. Clin Cancer Res 9(5):1611-1615

19. Kawakami K et al (2001) Methylenetetrahydrofolate reductase polymorphism is associated with folate pool in gastrointestinal cancer tissue. Anticancer Res 21(1a):285-289

20. Dahl O et al (2009) Final results of a randomised phase III study on adjuvant chemotherapy with $5 \mathrm{FU}$ and levamisol in colon and rectum cancer stage II and III by the Norwegian Gastrointestinal Cancer Group. Acta Oncol 48(3):368-376

21. Petersen SH et al (2012) Postoperative adjuvant chemotherapy in rectal cancer operated for cure. Cochrane Database Syst Rev 3:4078

22. Sadahiro S et al (2010) Molecular determinants of folate levels after leucovorin administration in colorectal cancer. Cancer Chemother Pharmacol 65(4):735-742

23. Carlsson G et al (1995) Antitumour effects of pure diastereoisomers of 5-formyltetrahydrofolate in hepatic transplants of a rodent colon carcinoma model. Biochem Pharmacol 50(9):1347-1351

24. Kim YI et al (1998) Colonic mucosal concentrations of folate correlate well with blood measurements of folate status in persons with colorectal polyps. Am J Clin Nutr 68(4):866-872 